\title{
Mean square value of exponential sums related to representation of integers as sum of two squares
}

by

Pavel M. Bleher and Freeman J. Dyson (Princeton, N.J.)

1. Introduction. The problem we address here arises in the study of the error function in the shifted circle problem (see [BCDL]). Let $\alpha=\left(\alpha_{1}, \alpha_{2}\right) \in$ $\mathbb{R}^{2}$ be a fixed point in a plane. Define

$$
N(R ; \alpha)=\#\left\{m \in \mathbb{Z}^{2}:|m-\alpha| \leq R\right\}
$$

and

$$
F(R ; \alpha)=\frac{N(R ; \alpha)-\pi R^{2}}{R^{1 / 2}} .
$$

A long-standing famous conjecture of Hardy (see $[\mathrm{H}]$ ) is to prove that when $R \rightarrow \infty$,

$$
F(R ; \alpha)=O\left(R^{\varepsilon}\right), \quad \forall \varepsilon>0
$$

(Hardy considered $\alpha=0$ ). In [BCDL] and [B] it was proved that the mean square limit

$$
D(\alpha)=\lim _{T \rightarrow \infty} \frac{1}{T} \int_{1}^{T}|F(R ; \alpha)|^{2} d R
$$

exists and is equal to

$$
D(\alpha)=\left(2 \pi^{2}\right)^{-1} \sum_{n=1}^{\infty} n^{-3 / 2}\left|r_{\alpha}(n)\right|^{2},
$$

where

$$
r_{\alpha}(n)=\sum_{k^{2}+l^{2}=n} e\left(\alpha_{1} k+\alpha_{2} l\right), \quad e(t)=\exp (2 \pi i t)
$$

(for $\alpha=0$ this reduces to a classical result of Cramér $[\mathrm{C}]$ ). The existence of a limit distribution $p_{\alpha}(t) d t$ of $F(R ; \alpha)$ was shown in [BCDL] as well:

$$
\lim _{t \rightarrow \infty} \frac{1}{T} \int_{\{R: 1 \leq R \leq T, a \leq F(R ; \alpha) \leq b\}} d R=\int_{a}^{b} p_{\alpha}(t) d t
$$


for every $a<b$ (for $\alpha=0$ this result is due to Heath-Brown, see [H-B]). The density $p_{\alpha}(t)$ was proved to be an analytic function in $t$ which decays at infinity, roughly speaking, as $C \exp \left(-\lambda t^{4}\right)$.

In [BCDL] one of the key points in the proof was to evaluate the asymptotics of the series

$$
S_{\alpha}(b)=\sum_{n=1}^{\infty}\left|r_{\alpha}(n)\right|^{2} \exp (-n / b)
$$

when $b \rightarrow \infty$. This gives a mean square value of $\left|r_{\alpha}(n)\right|$ as $n \rightarrow \infty$. In the present work we show that $S_{\alpha}(b)$ has an unexpected wild behavior. Namely, $S_{\alpha}(b)$, as a function of $\alpha$, has a "bumpy" shape when $b \rightarrow \infty$, with a big bump at every rational point $\alpha$. This behavior of $S_{\alpha}(b)$ is closely related to the fact, discovered in [BD], that the mean square limit $D(\alpha)$ has a sharp local maximum at every rational point. We prove here the following theorems:

Theorem 1.1. For any fixed $\alpha$,

$$
\liminf _{b \rightarrow \infty}\left(b^{-1} S_{\alpha}(b)\right) \geq \pi .
$$

TheOREM 1.2. Except for an exceptional set of $\alpha$ of measure zero in $\mathbb{R}^{2}$,

$$
S_{\alpha}(b)=\pi b+O\left(b^{3 / 4+\varepsilon}\right) \quad \text { as } b \rightarrow \infty .
$$

Re mark. We prove in Theorem 1.5 that all rational $\alpha$ and all $\alpha$ sufficiently rapidly approximable by rationals belong to the exceptional set. Theorem 1.3 implies the weaker statement that

$$
\lim _{b \rightarrow \infty}\left(b^{-1} S_{\alpha}(b)\right)=\pi
$$

for almost all $\alpha$. The power $3 / 4$ in Theorem 1.2 is best possible by virtue of (3.13) below.

Theorem 1.3. Assume that $\alpha=\left(\alpha_{1}, \alpha_{2}\right)$ is Diophantine, i.e.,

$$
\left|\alpha_{1} k+\alpha_{2} l-n\right|>C\left(k^{2}+l^{2}\right)^{-D}
$$

with some $C, D>0$ for all integers $k, l, n$ with $k^{2}+l^{2} \neq 0$. Then (1.4) holds.

TheOrem 1.4. Assume that

$$
\alpha_{1} p+\alpha_{2} q-r=0
$$

for some integer $p, q, r$ with coprime $p, q \neq 0$. Assume also that $\alpha_{1}$ is Diophantine, i.e.,

$$
\left|k \alpha_{1}-l\right|>C\left(k^{2}\right)^{-D},
$$

with some $C, D>0$ for all integers $k, l$ with $k \neq 0$. Then

$$
\lim _{b \rightarrow \infty}\left(b^{-1} S_{\alpha}(b)\right)=\pi\left(1+\varepsilon(p q)\left(p^{2}+q^{2}\right)^{-1}\right),
$$


where

$$
\varepsilon(n)= \begin{cases}1 & \text { if } n \text { is even, } \\ 2 & \text { if } n \text { is odd. }\end{cases}
$$

R e m a rk. Our proof shows that (1.6) without the assumption that either $\alpha_{1}$ or $\alpha_{2}$ is Diophantine implies

$$
\liminf _{b \rightarrow \infty}\left(b^{-1} S_{\alpha}(b)\right) \geq \pi\left(1+\varepsilon(p q)\left(p^{2}+q^{2}\right)^{-1}\right) .
$$

THEOREM 1.5. Suppose the vector $\alpha$ is rational, i.e., there exists an integer $Q$ such that

$$
2 Q \alpha_{1}=n_{1}, \quad 2 Q \alpha_{2}=n_{2}
$$

are integers and $\operatorname{gcd}\left(Q, n_{1}, n_{2}\right)=1$. Then

$$
(b \log b)^{-1} S_{\alpha}(b)=C(Q r(Q))^{-1}+O\left(\log ^{-1} b\right) \quad \text { as } b \rightarrow \infty,
$$

$$
r(Q)=\prod_{p \mid Q}\left(1+p^{-1}\right),
$$

with the product taken over primes $p$ dividing $Q$, and

$$
\begin{aligned}
& C=3(Q \text { even }), \quad C=4\left(Q \text { odd }, n_{1}+n_{2} \text { even }\right), \\
& C=2\left(Q \text { odd }, n_{1}+n_{2} \text { odd }\right) .
\end{aligned}
$$

Re mark. We have $Q<\operatorname{Qr}(Q) \leq \sigma(Q)$, where $\sigma(Q)$ is the sum of the divisors of $Q$. According to Theorem 323 in [HW],

$$
\sigma(n)=O(n \log \log n) .
$$

Corollary 1. For fixed rational $\alpha$, the mean-square value of $r_{\alpha}(n)$ for $n$ of the order of magnitude $N$ is at least $2(\sigma(Q))^{-1} \log (N / Q)$.

COROLlaRY 2. If $\alpha$ is an almost-rational vector, i.e., if an infinite sequence $\left\{Q_{1}, Q_{2}, \ldots\right\}$ of integers exists such that

$$
2 Q_{j} \alpha=P_{j}+\varepsilon_{j},
$$

with $P_{j}$ integral vectors and

$$
\left(\sigma\left(Q_{j}\right)\right)^{-1} \log \left(\left|\varepsilon_{j}\right|^{-1}\right) \rightarrow \infty \quad \text { as } j \rightarrow \infty,
$$

then

$$
\limsup _{b \rightarrow \infty}\left(b^{-1} S_{\alpha}(b)\right)=\infty .
$$

The Tauberian theorem of Hardy and Littlewood (see [HL]) enables us to derive from Theorems 1.2-1.5 the asymptotics of

$$
\sigma_{\alpha}(n)=n^{-1} \sum_{k=1}^{n}\left|r_{\alpha}(k)\right|^{2}
$$

as $n \rightarrow \infty$. The theorem of Hardy and Littlewood is the following: 
THEOREM HL. If $f(x)=\sum a_{n} x^{n}$ is a power series with positive coefficients, and

$$
f(x) \sim A(1-x)^{-1}|\log (1-x)|^{\alpha} \quad(x \rightarrow 1),
$$

where $A>0$ and $\alpha \geq 0$, then

$$
a_{1}+\ldots+a_{n} \sim A n \log ^{\alpha} n .
$$

Define $a_{n}=\left|r_{\alpha}(n)\right|^{2}, 1-x=\exp (-b)$. Then we see from Theorems $1.2-1.5$ and HL that for all Diophantine $\alpha$,

$$
\lim _{n \rightarrow \infty} \sigma_{\alpha}(n)=\pi
$$

for all $\alpha$ satisfying (1.6), (1.7),

$$
\lim _{n \rightarrow \infty} \sigma_{\alpha}(n)=\pi\left(1+\varepsilon(p q)\left(p^{2}+q^{2}\right)^{-1}\right) ;
$$

and finally for all rational $\alpha$,

$$
\lim _{n \rightarrow \infty}(\log n)^{-1} \sigma_{\alpha}(n)=C(Q r(Q))^{-1},
$$

with $r(Q)$ and $C$ defined in (1.12) and (1.13), respectively.

Theorems 1.1 and 1.5 were proved in [BCDL]. Here we prove Theorems 1.2-1.4 and Corollary 2 of Theorem 1.5.

2. Preliminaries from [BCDL]. Here we recall some results from [BCDL]. The sum (1.1) may be written

$$
S_{\alpha}(b)=\sum_{m, m^{\prime}} e\left(\alpha\left(m-m^{\prime}\right)\right) \exp \left(-m^{2} / b\right),
$$

summed over integer vectors $m, m^{\prime} \in \mathbb{Z}^{2} \backslash\{0\}$ with $m^{2}=m^{\prime 2}$. As was shown in [BCDL], the sum (2.1) can be converted into an unrestricted sum,

$$
S_{\alpha}(b)=\frac{1}{2} \sum_{k, l, j, h} e\left(h\left(l \alpha_{1}-k \alpha_{2}\right)\right) \exp \left(-\left(k^{2}+l^{2}\right)\left(j^{2}+h^{2}\right) /(4 b)\right),
$$

summed over all $(j, k, l, h) \in \mathbb{Z}^{4}$ satisfying

$$
h^{2}+j^{2} \neq 0, \quad k^{2}+l^{2} \neq 0,
$$$$
\text { either } j \equiv h \equiv 0 \text {, or } j \equiv h \equiv k \equiv l \equiv 1(\bmod 2) \text {, }
$$

and

$$
k, l \text { are relatively prime, }
$$

which means that either $|k|+|l|=1$, or $\operatorname{gcd}(|k|,|l|)=1$.

According to the two possibilities in $(2.4)$ we divide $S_{\alpha}(b)$ into even and odd parts,

$$
S_{\alpha}(b)=S_{\mathrm{e}}+S_{\mathrm{o}}
$$


where the terms with $j$ and $h$ even are

$$
S_{\mathrm{e}}=\frac{1}{2} \sum_{k, l}[F(w) F(0)-1],
$$

summed over integers $(k, l)$ satisfying $(2.5)$, and

$$
S_{\mathrm{o}}=\frac{1}{2} \sum_{k, l} G(w) G(0),
$$

summed over odd integers $k$ and $l$ satisfying $(2.5)$. The functions $(F, G)$ are defined by

$$
\sum_{x} \exp \left(-x^{2} / a\right) e(x t)=F(t) \text { or } G(t),
$$

where the sum is over integer $x$ for $F$ and over half-odd-integer $x$ for $G$. In (2.7)-(2.9) we have used the abbreviations $w=2\left(l \alpha_{1}-k \alpha_{2}\right), a=b\left(k^{2}+\right.$ $\left.l^{2}\right)^{-1}$. By the Poisson summation formula, (2.9) gives

$$
\begin{aligned}
& F(t)=(\pi a)^{1 / 2} \sum_{p} \exp \left(-\pi^{2} a(p+t)^{2}\right), \\
& G(t)=(\pi a)^{1 / 2} \sum_{p}(-1)^{p} \exp \left(-\pi^{2} a(p+t)^{2}\right) .
\end{aligned}
$$

According to (2.9), the functions $F$ and $G$ are periodic with periods 1 and 2 respectively,

$$
F(w+1)=F(w), \quad G(w+1)=-G(w) .
$$

For $a \leq 1,(2.9)$ gives

$$
F(w)=1+O\left(\exp \left(-a^{-1}\right)\right), \quad G(w)=O\left(\exp \left(-(4 a)^{-1}\right)\right) .
$$

For $a \geq 1,(2.10)$ gives

$$
\begin{aligned}
& F(w)=(\pi a)^{1 / 2}\left[\exp \left(-\pi^{2} a \widehat{w}^{2}\right)+O\left(\exp \left(-(\pi / 2)^{2} a\right)\right)\right], \\
& G(w)=(-1)^{w}(\pi a)^{1 / 2}\left[\exp \left(-\pi^{2} a \widehat{w}^{2}\right)+O\left(\exp \left(-(\pi / 2)^{2} a\right)\right)\right],
\end{aligned}
$$

where $\widehat{w}$ is the distance of $w$ from the nearest integer.

3. Proof of Theorem 1.2. For Theorem 1.2 we divide the sum (2.1) into two parts

$$
S_{\alpha}(b)=I(b)+R_{\alpha}(b),
$$

where $I(b)$ consists of the terms with

$$
m=m^{\prime},
$$


which are equal to the terms in (2.2) with $h=0$. By (2.9) and (2.10),

$$
I(b)=\left(\sum_{x} \exp \left(-x^{2} / b\right)\right)^{2}=\pi b+O\left(b \exp \left(-\pi^{2} b\right)\right) .
$$

By (3.1) and (3.3), Theorem 1.2 states that

$$
R_{\alpha}(b)=O\left(b^{3 / 4+\varepsilon}\right)
$$

except for a set of $\alpha$ of measure zero.

Consider the integral

$$
J(b)=\int\left|R_{\alpha}(b)\right|^{2} d \alpha,
$$

integrated over the square

$$
0<\alpha_{1}<1, \quad 0<\alpha_{2}<1 .
$$

We represent $R_{\alpha}(b)$ by the sum $(2.2)$ with the condition $(h \neq 0)$ replacing (2.3). It is convenient to restrict the sum to positive $h$ and drop the factor $1 / 2$. When (2.2) is inserted into (3.5), the result is an eight-fold sum over the integers $\left(k, l, j, h, k^{\prime}, l^{\prime}, j^{\prime}, h^{\prime}\right)$. The integration over (3.6) eliminates all terms except those with

$$
h l=h^{\prime} l^{\prime}, \quad h k=h^{\prime} k^{\prime} .
$$

Since $h$ and $h^{\prime}$ are positive and the fractions $k / l$ and $k^{\prime} / l^{\prime}$ are reduced to their lowest terms by (2.5), (3.7) implies

$$
h=h^{\prime}, \quad k=k^{\prime}, \quad l=l^{\prime} .
$$

The eight-fold sum collapses to a five-fold sum

$$
J(b)=\sum_{k, l, h, j, j^{\prime}} \exp \left[-\left(k^{2}+l^{2}\right)\left(2 h^{2}+j^{2}+j^{\prime 2}\right) /(4 b)\right],
$$

with summations restricted only by

(3.10) $(k, l)=1, \quad h>0, \quad$ either $\left(j, j^{\prime}, h\right)$ all even or $\left(j, j^{\prime}, h, k, l\right)$ all odd.

When $b$ is large, each of the sums over $j$ and $j^{\prime}$ gives

$$
\left[\pi b /\left(k^{2}+l^{2}\right)\right]^{1 / 2}+O(1),
$$

and the sum over $h$ gives the same result multiplied by $2^{-3 / 2}$. Therefore (3.9) becomes

$$
J(b)=2^{-3 / 2} \sum_{k, l}\left(c_{k}+c_{l}\right)\left[\pi b /\left(k^{2}+l^{2}\right)\right]^{3 / 2}+O(b),
$$

where $c_{k}=0$ for $k$ even and $c_{k}=1$ for $k$ odd. The sum over $(k, l)$ is convergent, so that

$$
J(b)=B b^{3 / 2}+O(b)
$$


where $B$ is a calculable constant, namely

$$
B=(2 \pi)^{3 / 2}((3+\sqrt{2}) / 7) \zeta(3 / 2) L(3 / 2) / \zeta(3),
$$

where $\zeta$ and $L$ are the Riemann and Dirichlet functions,

$$
\zeta(s)=\sum_{n} n^{-s}, \quad L(s)=\sum_{n}(-1)^{n-1}(2 n-1)^{-s} .
$$

We need to prove from (3.5) and (3.13) that (3.4) holds except for a set of $\alpha$ of measure zero. But (3.4) does not follow from (3.13) alone. We need in addition the fact that $R_{\alpha}(b)$ is a smoothly-varying function of $b$, so that it cannot become large at isolated peaks without violating (3.13). To prove (3.4) we require bounds on all the derivatives of $R_{\alpha}(b)$. It is convenient to use the notations

$$
\begin{aligned}
D & =b^{-2}(d / d b), \\
J_{p}(b) & =\int\left|D^{p} R_{\alpha}(b)\right|^{2} d \alpha,
\end{aligned}
$$

integrated over (3.6). The same analysis that led to (3.9) now gives

$$
\begin{aligned}
J_{p}(b)=4^{-2 p} \sum_{k, l, h, j, j^{\prime}} \exp \left[-\left(k^{2}+l^{2}\right)\left(2 h^{2}+j^{2}+{j^{\prime}}^{2}\right) /(4 b)\right] \\
\times\left(h^{2}+j^{2}\right)^{p}\left(h^{2}+{j^{\prime}}^{2}\right)^{p}\left(k^{2}+l^{2}\right)^{2 p} .
\end{aligned}
$$

The sums over $\left(h, j, j^{\prime}\right)$ give

$$
A_{p}\left(b /\left(k^{2}+l^{2}\right)\right)^{2 p+3 / 2},
$$

plus terms of lower order in $b$, with a numerical constant $A_{p}$. Inserting (3.19) into (3.18) gives

$$
J_{p}(b)=A_{p} b^{2 p+3 / 2} \sum_{k, l}\left(k^{2}+l^{2}\right)^{-3 / 2}=B_{p} b^{2 p+3 / 2}+O\left(b^{2 p+1}\right) .
$$

Thus $D^{p} R_{\alpha}(b)$ has the root-mean-square order of magnitude

$$
b^{p+3 / 4} \text {. }
$$

We have to prove that this same order of magnitude estimate holds pointwise, for almost all $\alpha$, as $b \rightarrow \infty$ for fixed $\alpha$.

We use an induction on $p$, working downward from $p+1$ to $p$. Our inductive hypothesis says that

$$
\left|D^{p} R_{\alpha}(b)\right|<A b^{p+3 / 4+f(p)},
$$

with some positive $f(p)$ depending only on $p$, with $A$ depending on $p$ and $\alpha$ but not on $b$, except for a set of $\alpha$ of measure zero. We assume that (3.22) holds for $p+1$ and find for which $f(p)$ it will hold for $p$. Let $\left(b_{1}, b_{2}, \ldots\right)$ be a sequence of numbers tending to infinity, for example

$$
b_{j}=j^{m},
$$


with an exponent $m$ to be chosen later, such that

$$
\left|b_{j+1}-b_{j}\right|<A b_{j}^{1-1 / m} \text {. }
$$

The inductive hypothesis together with (3.24) implies that for every $b$ in the range

$$
b_{j} \leq b<b_{j+1},
$$

we have

$$
\left|D^{p} R_{\alpha}(b)-D^{p} R_{\alpha}\left(b_{j}\right)\right|<A b^{p+3 / 4+f(p+1)-1 / m} .
$$

Comparing (3.26) with (3.22), we see that if

$$
f(p+1)<f(p)+1 / m
$$

then (3.22) holds for all $b$ if and only if

$$
\left|D^{p} R_{\alpha}\left(b_{j}\right)\right|<A b_{j}^{p+3 / 4+f(p)}
$$

holds for all $j$ and some $A$ depending on $\alpha$, with the usual exception of a set of $\alpha$ of measure zero. Therefore, to complete the induction it is only necessary to prove (3.28).

Let $m_{j p}(A)$ be the measure of the set of $\alpha$ for which (3.28) is false for a particular $j$. Comparing (3.28) with (3.17) and (3.20), we see that

$$
m_{j p}(A)<B_{p} A^{-2} b_{j}^{-2 f(p)}\left(1+O\left(b_{j}^{-1 / 2}\right)\right) .
$$

Therefore

$$
\sum_{j} m_{j p}(A)<C_{p} A^{-2},
$$

where $C_{p}$ is the sum of the coefficients on the right of (3.29). The series (3.30) converges and the sum is finite by (3.23) if

$$
1 / m<2 f(p) .
$$

The left side of (3.30) is an upper bound to the measure of the set of $\alpha$ for which (3.28) is false for a given $A$ and at least one $j$. The set of $\alpha$ for which (3.28) is false for every $A$ and some $j$ has measure less than (3.30) for every $A$, i.e. has measure zero. So we have proved that (3.28) holds for almost all $\alpha$ if (3.31) holds. We proved before that (3.22) follows from (3.28) if (3.27) holds. Thus the induction of the hypothesis (3.22) from $p+1$ to $p$ succeeds, provided that we can satisfy both (3.27) and (3.31) with the same $m$. This will be possible if and only if

$$
f(p+1)<3 f(p) .
$$

To start the induction we use the estimate

$$
\left|D^{p} R_{\alpha}(b)\right|<\sum_{n} n^{p}\left|r_{0}(n)\right|^{2} \exp (-n / b)=O\left(b^{p+1+\varepsilon}\right),
$$


which follows from

$$
\left|r_{\alpha}(n)\right| \leq r_{0}(n)=O\left(n^{\varepsilon}\right) .
$$

Choose any integer $P$. The inductive hypothesis (3.22) holds for $p=P$ by (3.33) if

$$
f(P)>1 / 4 \text {. }
$$

The induction requires only that (3.32) hold for $p<P$, which is true if we take

$$
f(p)=K^{p-P},
$$

with any constant $K<3$. So the induction is complete and proves (3.22) with $f(p)$ given by (3.36), for any value of $P$. But the choice of $P$ is arbitrary. We can let $P \rightarrow \infty$ in (3.36) and deduce that (3.22) holds for any $p$ provided that

$$
f(p)>0 .
$$

In particular, when $p=0,(3.22)$ with (3.37) implies (3.4), and Theorem 1.2 is proved.

\section{Proof of Theorems 1.3 and $\mathbf{1 . 4}$}

Proof of Theorem 1.3. By (2.6), $S_{\alpha}(b)=S_{\mathrm{e}}+S_{\mathrm{o}}$. Following [BCDL] we divide $S_{\mathrm{e}}$ into two parts, $S_{\mathrm{e} 1}+S_{\mathrm{e} 2}$, with $\widehat{w}>\delta$ and with $\widehat{w} \leq \delta$, respectively, where $\delta>0$ is an arbitrary small number. Similar division is defined for $S_{\mathrm{o}}$. (B.75), (B.77) in [BCDL] prove

$$
\lim _{\delta \rightarrow 0} \limsup _{b \rightarrow \infty} b^{-1}\left|S_{1}-\pi\right|=0,
$$

where $S_{1}=S_{\mathrm{e} 1}+S_{\mathrm{o} 1}$. Therefore Theorem 1.3 will be proved if we prove for $S_{2}=S_{\mathrm{e} 2}+S_{\mathrm{o} 2}$ the following result:

Lemma 4.1. Assume that $\alpha=\left(\alpha_{1}, \alpha_{2}\right)$ is Diophantine, i.e., (1.5) holds. Then

$$
\lim _{\delta \rightarrow 0} \limsup _{b \rightarrow \infty} b^{-1}\left|S_{2}\right|=0 .
$$

Proof. We shall estimate $S_{\mathrm{e} 2} ; S_{\mathrm{o} 2}$ can be estimated in the same way. We start with the definition of $S_{\mathrm{e} 2}$ :

$$
S_{\mathrm{e} 2}=\frac{1}{2} \sum_{k, l}[F(w) F(0)-1]
$$

with the summation over $k, l$ with $(k, l)=1$ and $\widehat{w} \leq \delta$. Let us divide $S_{\mathrm{e} 2}$ into four parts, $S_{\mathrm{e} 2}=S_{3}+S_{4}+S_{5}+S_{6}$, where

$$
S_{j}=\frac{1}{2} \sum_{M_{j}}[F(w) F(0)-1]
$$


with

$$
\begin{aligned}
& M_{3}=\left\{k, l:(k, l)=1 ; \widehat{w} \leq \delta ; a \leq|\log \delta|^{-2}\right\} \\
& M_{4}=\left\{k, l:(k, l)=1 ; \widehat{w} \leq \delta ;|\log \delta|^{-2}<a \leq \delta^{-1 / 3}\right\} ; \\
& M_{5}=\left\{k, l:(k, l)=1 ; \widehat{w} \leq \delta ; \delta^{-1 / 3}<a ; \exp \left(-\pi^{2} a \widehat{w}^{2}\right) \leq a^{-1}\right\} ; \\
& M_{6}=\left\{k, l:(k, l)=1 ; \widehat{w} \leq \delta ; \delta^{-1 / 3}<a ; \exp \left(-\pi^{2} a \widehat{w}^{2}\right)>a^{-1}\right\} .
\end{aligned}
$$

Now we shall estimate in turn $S_{3}, \ldots, S_{6}$. Without loss of generality we may assume that the summation in $k, l$ goes over the region $|l| \geq|k|$, because the sum over the complementary set $|l|<|k|$ can be estimated in the same way.

In $M_{3}, a$ is small, so by $(2.13)$,

$$
|F(w) F(0)-1| \leq C \exp \left(-a^{-1}\right)=C \exp \left(-\left(k^{2}+l^{2}\right) / b\right),
$$

hence

$$
\left|S_{3}\right| \leq C \sum_{k^{2}+l^{2} \geq b|\log \delta|^{2}} \exp \left(-\left(k^{2}+l^{2}\right) / b\right) \leq C_{0} b \exp \left(-|\log \delta|^{2}\right),
$$

which satisfies (4.2).

From (2.13), (2.14), $|F(w) F(0)-1| \leq C a$, hence

$$
\left|S_{4}\right| \leq C \delta^{-1 / 3} \sum_{M_{4}} 1 .
$$

By (B.48), (B.49) in [BCDL], for every fixed $k$ the fraction of $l$ with $\widehat{w}<\delta$ does not exceed $2 \delta+4 / N$, hence

$$
\begin{aligned}
\sum_{M_{4}} 1 & \leq(2 \delta+4 / N) \sum_{a \leq \delta^{-1 / 3}} 1=(2 \delta+4 / N) \sum_{k^{2}+l^{2} \leq b \delta^{-1 / 3}} 1 \\
& \leq C(2 \delta+4 / N) b \delta^{-1 / 3} .
\end{aligned}
$$

Hence

$$
\left|S_{4}\right| \leq C_{0} b(2 \delta+4 / N) \delta^{-2 / 3} .
$$

Since we can take $N \rightarrow \infty$ as $b \rightarrow \infty, S_{4}$ also satisfies (4.2).

In $M_{5}$, by $(2.14),|F(w)| \leq C a^{1 / 2} \exp \left(-\pi^{2} a \widehat{w}^{2}\right) \leq C_{0} a^{-1 / 2}$, hence

$$
|F(w) F(0)-1| \leq C_{1},
$$

and

$$
\left|S_{5}\right| \leq C_{1} \sum_{a \geq \delta^{-1 / 3}} 1=C_{1} \sum_{k^{2}+l^{2} \leq b \delta^{1 / 3}} 1 \leq C_{2} b \delta^{1 / 3} .
$$

Thus $S_{5}$ satisfies (4.2). 
In $M_{6}$, $\exp \left(-\pi^{2} a \widehat{w}^{2}\right)>a^{-1}$, hence $\pi^{2} a \widehat{w}^{2}<\log a$, and

$$
\widehat{w}<\pi^{-1}\left(a^{-1} \log a\right)^{1 / 2} .
$$

Therefore $\widehat{w}$ small for large $a$. Due to the Diophantine condition this implies that for some $\zeta>0$ in the circle

$$
k^{2}+l^{2} \leq b^{\zeta}
$$

there is no point from $M_{6}$. Indeed, in $M_{6}$, due to (1.5) and (4.3),

$$
C\left(k^{2}+l^{2}\right)^{-D} \leq \widehat{w} \leq \pi^{-1}\left(\left(k^{2}+l^{2}\right) / b\right)^{1 / 2}\left|\log \left(\left(k^{2}+l^{2}\right) / b\right)\right|^{1 / 2} .
$$

This implies that for large $b$,

$$
k^{2}+l^{2}>b^{\zeta}
$$

with $\zeta=(2 D+1)^{-1}+\varepsilon, \varepsilon>0$, hence in the circle (4.4) there is no point from $M_{6}$.

Let us divide $M_{6}$ into annular parts $M_{6 j}=M_{6} \cap A_{j}$ with

$$
A_{j}=\left\{2^{j-1} \delta^{-1 / 3}<a \leq 2^{j} \delta^{-1 / 3}\right\}=\left\{2^{-j} \delta^{1 / 3} b \leq k^{2}+l^{2}<2^{-j+1} \delta^{1 / 3} b\right\},
$$

$j=1, \ldots, J$, where $J$ is the least integer number with $2^{-J} \delta^{1 / 3} b<b^{\zeta}$. Let us fix some $j, 1 \leq j \leq J$, and estimate

$$
S_{6 j}=\sum_{M_{6 j}}|F(w) F(0)-1| \leq C a\left|M_{6 j}\right|
$$

where $a=b /\left(k^{2}+l^{2}\right)$ refers to an arbitrary point inside $M_{6 j}$.

Let $s$ be the width of the annulus $A_{j}$. For $(k, l) \in A_{j}$,

$$
C_{0} s<\left(k^{2}+l^{2}\right)^{1 / 2}<C_{1} s .
$$

For a fixed $k$, the number of $l$ with $\widehat{w}<\lambda=\pi^{-1}\left(a^{-1} \log a\right)^{1 / 2}$ is estimated by (see (B.47) of $[$ BCDL])

$$
(s / N+1)(\lambda N+2)=\lambda s+\lambda N+s / N+2,
$$

where $N<s$ is the denominator of an approximant $M / N$ of $2 \alpha_{2}$. So

$$
\left|M_{6 j}\right| /\left|A_{j}\right| \leq C(\lambda+1 / N)
$$

and

$$
\left|S_{6 j}\right| \leq C \sum_{A_{j}} a(\lambda+1 / N) .
$$

Let $N_{i} \leq s<N_{i+1}$, where $N_{i}$ are the denominators of subsequent approximants. The Diophantine condition implies

$$
C N_{i}^{-D} \leq\left|M_{i} / N_{i}-\alpha_{2}\right| \leq\left|M_{i} / N_{i}-M_{i+1} / N_{i+1}\right|=\left(N_{i} N_{i+1}\right)^{-1},
$$


hence $N_{i} \geq\left(C N_{i+1}\right)^{(D-1)^{-1}} \geq(C s)^{(D-1)^{-1}}$ and $N_{i}^{-1} \leq C_{0} s^{-(D-1)^{-1}}$. Therefore from (4.7),

$$
\left|S_{6 j}\right| \leq C \sum_{A_{j}} a\left(\lambda+s^{-\gamma}\right)
$$

with $\gamma=(D-1)^{-1}$. Hence

$$
\left|S_{6 j}\right| \leq C_{0} \sum_{A_{j}} a\left(\left(a^{-1} \log a\right)^{1 / 2}+\left(k^{2}+l^{2}\right)^{-\gamma / 2}\right)
$$

or

$$
\left|S_{6}\right| \leq C_{0} \sum_{(1 / 2) b^{-\zeta} \leq k^{2}+l^{2} \leq b \delta^{1 / 3}} a\left(\left(a^{-1} \log a\right)^{1 / 2}+\left(k^{2}+l^{2}\right)^{-\gamma / 2}\right) .
$$

Now,

$$
\begin{aligned}
\sum_{k^{2}+l^{2} \leq b \delta^{1 / 3}}(a \log a)^{1 / 2} & =\sum_{k^{2}+l^{2} \leq b \delta^{1 / 3}}\left(b /\left(k^{2}+l^{2}\right)\right)^{1 / 2} \log ^{1 / 2}\left(b /\left(k^{2}+l^{2}\right)\right) \\
& \leq C \delta^{-1 / 6} \log ^{1 / 2} \delta^{-1 / 3} b \delta^{1 / 3}=(C / 3) b \delta^{1 / 6}|\log \delta|^{1 / 2}
\end{aligned}
$$

and

$$
\sum_{(1 / 2) b^{-\zeta} \leq k^{2}+l^{2}} a\left(k^{2}+l^{2}\right)^{-\gamma / 2}=\sum_{(1 / 2) b^{-\zeta} \leq k^{2}+l^{2}} b\left(k^{2}+l^{2}\right)^{-1-\gamma / 2} \leq C b^{1-\zeta \gamma},
$$

which implies

$$
\left|S_{6}\right| \leq C b\left(\delta^{1 / 6}|\log \delta|^{1 / 2}+b^{-\zeta \gamma}\right) .
$$

Therefore $S_{6}$ satisfies (4.2), and Lemma 4.1 is proved.

Proof of Theorem 1.4. In virtue of (4.1), Theorem 1.4 will be proved if we prove the following lemma:

Lemma 4.2. Assume that $\alpha=\left(\alpha_{1}, \alpha_{2}\right)$ satisfies (1.6), (1.7). Then

$$
\lim _{\delta \rightarrow 0} \limsup _{b \rightarrow \infty}\left|b^{-1} S_{2}-\varepsilon(p q)\left(p^{2}+q^{2}\right)\right|=0,
$$

with $\varepsilon(n)=(n \bmod 2)+1$.

Proof. The proof of Lemma 4.2 repeats word for word the one of Lemma 4.1 excepting one point: we proved in Lemma 4.1 that if $\alpha$ is Diophantine then in the circle (4.4) there is no point from $M_{6}$; now we state that if $\alpha$ satisfies (1.6), (1.7) then in the circle (4.4) there are exactly two points from $M_{6}$,

$$
(k, l)= \pm(-q, p) .
$$

Notice that due to (1.6), if (4.9) holds then

$$
w=2\left(l \alpha_{1}-k \alpha_{2}\right)= \pm 2\left(p \alpha_{1}+q \alpha_{2}\right)= \pm 2 r,
$$


hence $\widehat{w}=0$, so that these two points contribute to $S_{\mathrm{e}}$ the term

$$
F^{2}(0)-1=\pi b\left(k^{2}+l^{2}\right)^{-1}+O(1)=\pi b\left(p^{2}+q^{2}\right)^{-1}+O(1) .
$$

If $p q$ is odd, then these two points contribute a similar term to $S_{\mathrm{o}}$. Therefore totally they contribute to $S_{\alpha}(b)$ the term $\pi b \varepsilon(p q)\left(p^{2}+q^{2}\right)^{-1}+O(1)$.

These considerations show that (4.8) will be proved if we prove that (4.9) are the only points from $M_{6}$ in the circle (4.4). Without loss of generality we may assume $p \neq 0$. Assume

$$
(k, l) \neq \pm(-q, p) .
$$

We have

(4.11) $l \alpha_{1}-k \alpha_{2}=(l / p)\left(\alpha_{1} p+\alpha_{2} q\right)-\alpha_{2}(k+l q / p)=(l / p) r-\alpha_{2}(k+l q / p)$.

Note that

$$
k+l q / p \neq 0 .
$$

Indeed, otherwise $l q=-k p$, and since the pairs $(k, l)$ and $(p, q)$ are coprime, $(k, l)= \pm(-q, p)$, which contradicts (4.10).

(4.11), (4.12) and (1.7) imply that for every integer $n$,

$$
\left|2 p\left(l \alpha_{1}-k \alpha_{2}\right)-n\right|=\left|-2 \alpha_{2}(k p+l q)+2 l r-n\right| \geq C(2|k p+l q|)^{-2 D},
$$

hence if $m$ is the closest integer to $w$, then

$$
p \widehat{w}=p\left|2\left(l \alpha_{1}-k \alpha_{2}\right)-m\right| \geq C(2|k p+l q|)^{-2 D} .
$$

Hence

$$
\widehat{w} \geq C_{p q}\left(k^{2}+l^{2}\right)^{-D} .
$$

This proves that (1.6), (1.7) and (4.10) imply (4.13). If we assume in addition that $(k, l) \in M_{6}$, then (4.5) holds. Since (4.5) implies (4.6), the point $(k, l)$ lies outside of the circle (4.4). This means that (4.9) are the only points from $M_{6}$ in this circle. Lemma 4.2 is proved.

Appendix. Proof of Corollary 2 of Theorem 1.5. The proof of Corollary 2 is the same as the proof of Theorem $1.5=$ Theorem B.3 in [BCDL], except that $w=2\left(l \alpha_{1}-k \alpha_{2}\right)$ is now an approximate integer instead of an exact integer when

$$
l P_{j 1}-k P_{j 2} \equiv 0 \bmod Q_{j} .
$$

From (2.14),

$$
F(w) F(0)-1>\pi a \exp (-1),
$$

with the particular choice of $b$ given by

$$
b=b_{j}=\pi\left|\varepsilon_{j}\right|^{-2}\left(Q_{j}\right)^{2} .
$$


Instead of (1.11) we now have

$$
S_{\alpha}\left(b_{j}\right)>C \exp (-1) b_{j}\left(Q_{j} r\left(Q_{j}\right)\right)^{-1} \log \left(b_{j} / Q_{j}\right)+O\left(b_{j}\right) .
$$

From (1.15), (A.3) and (A.4), (1.16) follows immediately.

Acknowledgements. This work is supported in part by a grant from the Ambrose Monell Foundation.

\section{References}

[B] P. M. Bleher, On the distribution of the number of lattice points inside a family of convex ovals, Duke Math. J. 67 (1992), 461-481.

[BCDL] P. M. Bleher, Z. Cheng, F. J. Dyson and J. L. Lebowitz, Distribution of the error term for the number of lattice points inside a shifted circle, Comm. Math. Phys. 154 (1993), 433-469.

[BD] P. M. Bleher and F. J. Dyson, The variance of the error function in the shifted circle problem is a wild function of the shift, Comm. Math. Phys., to appear.

[C] H. Cramér, Über zwei Sätze von Herrn G. H. Hardy, Math. Z. 15 (1922), 201-210.

[H] G. H. Hardy, The average order of the arithmetic functions $P(x)$ and $\Delta(x)$, Proc. London Math. Soc. 15 (1916), 192-213.

[HL] G. H. Hardy and J. E. Littlewood, Tauberian theorems concerning power series and Dirichlet series whose coefficients are positive, ibid. 13 (1914), 174.

[HW] G. H. Hardy and E. M. Wright, An Introduction to the Theory of Numbers, 4th ed., Oxford, 1960.

[H-B] D. R. Heath-Brown, The distribution and moments of the error term in the Dirichlet divisor problem, Acta Arith. 60 (1992), 389-415.

SCHOOL OF NATURAL SCIENCES

INSTITUTE FOR ADVANCED STUDY

PRINCETON, NEW JERSEY 08540

U.S.A. 